Information Sciences Letters

An International Journal

@ 2012 NSP

Natural Sciences Publishing Cor.

\title{
Indices to Quantify Arabic Journal Ranks and Research Output
}

\author{
M. Abdel-Aty \\ Center of Scientific Publications, University of Bahrain, 32038 Kingdom of Bahrain \\ Department of Mathematics, Sohag University, 82524 Sohag, Egypt \\ mabdelaty@uob.edu.bh
}

\begin{abstract}
We propose two simple indices to classify journals published in the Arabic language and different Arabic researchers. These indices depend on the known impact factor and h-index. The new indices give an easy way to judge the ranking of any journal (output of any researcher) without reference to other non-Arabic journals (output of non-Arabic researchers).

Keywords: Impact Factor, H-index
\end{abstract}

\section{Introduction}

Journal ranking is an important issue for institutions and researchers around the world. The existing tool of the impact factor allows users to configure their ranking interests, as well as provide a more reasonable method to evaluate a journal's impact. The impact factor (IF) is a measure of the frequency, in a particular year or other period of time, an average article in a journal has been cited. It is one of the evaluation tools provided by Thomson Reuters Journal Citation Reports JCR [1-3].

The annual Journal Citation Reports impact factor is a ratio between citations and recent citable items published: a journal's impact factor is calculated by dividing the number of current year citations to the source items published in that journal during the previous two years. The IF is used to compare different journals within a certain field. In a given year, the impact factor of a journal is the average number of citations received per paper published in that journal during the two preceding years [2]. For example, if a journal has an impact factor of 5 in 2012, then its papers published in 2010 and 2011 received 5 citations each on average in 2012. The 2012 impact factor of a journal would be calculated as follows:

$$
I F=A / B
$$

Here $A$ is the number of times articles published in 2010 and 2011 were cited by indexed journals during 2012 and B is the total number of "citable items" published by that journal in 2010 and 2011. ("citable items" are usually articles, reviews, proceedings, or notes; not editorials or Lettersto-the-Editor.)

New journals, which are indexed from their first published issue, will receive an impact factor after two years of indexing; in this case, the citations to the year prior to Volume 1, and the number of articles published in the year prior to Volume 1 are known to be zero values. Journals that are indexed starting with a volume other than the first volume will not get an impact factor 
until they have been indexed for three years. Annuals and other irregular publications sometimes publish no items in a particular year, affecting the count. The impact factor relates to a specific time period; it is possible to calculate it for any desired period, and the Journal Citation Reports (JCR) also includes a 5-year impact factor [3].

In 2005, J. E. Hirsch [4] proposed an index to quantify an individual's scientific research output which is called the h-index. Hirsch defined his index, as the number of papers with citation numbers higher or equal to h, i.e. a scientist has index $h$ if $h$ of his/her $N_{p}$ papers have at least $h$ citations each, and the other $\left(N_{p}-h\right)$ papers have no more than $h$ citations each. This index is used to characterize the scientific output of a researcher in a very good way. The way of calculation of the h-index includes the total number of papers published over a certain period of years and the number of citations for each paper. Now the ISI (Institute of Scientific Information) Web of Knowledge indexes more than 11,000 science and social science journals [3] and uses the impact factor to report on ranking the journals. Also, Scopus [5] uses the h-index to characterize the scientific output of a researcher.

Leo Egghe [6] has suggested what is called $g$-index. This index is used to quantifying scientific productivity based on publication records and is calculated based on the distribution of citations received by a given researcher's publications. Given a set of articles, ranked in decreasing order of the number of citations that they received, the $g$-index is the (unique) largest number such that the top $g$ articles received (together) at least $g^{2}$ citations. In agreement with the $h$-index, the $g$-index is a number close to the $h$-index for the same authors.

In this communication I introduce two alternative indices that can be used to estimate of the impact of Journals published in the Arabic Language as well as Arabic scientist's cumulative research contributions.

\section{Suggested Model}

The above methods have motivated the investigation of possible models to be applied to journals published in the Arabic language and researchers who compose their work in Arabic. This to avoid complications related to the use of the impact factor and h-index. On one hand, is the question of what kind of indications the impact factor ( $h$-index) gives. This question has already been answered [5] and it turns out to be a good indicator provided we know the impact factor ( $h$ index) of other journals, i.e., this number has no meaning if we do not know the other journals' (researchers') impact factor ( $h$-index). From this, it follows that in order to achieve the strongest indicators for any journal or researcher, one should think of building a model system capable of creating incoming percentage value.

For a journal's rank, I suggest to use the following model

$$
A F=\left(\frac{A}{A+B}\right) \times 100
$$

Here $A$ is the total number of times articles published in this journal in a preceding year were cited by indexed journals during the following year and B is the total number of "citable items" published by that journal in the preceding year. The application of this model shows that the results corresponding to the impact factor. However, here we can clearly know the impact of any 
journal without looking for the impact of other journals, since the result is a percentage between 0 and $100 \%$.

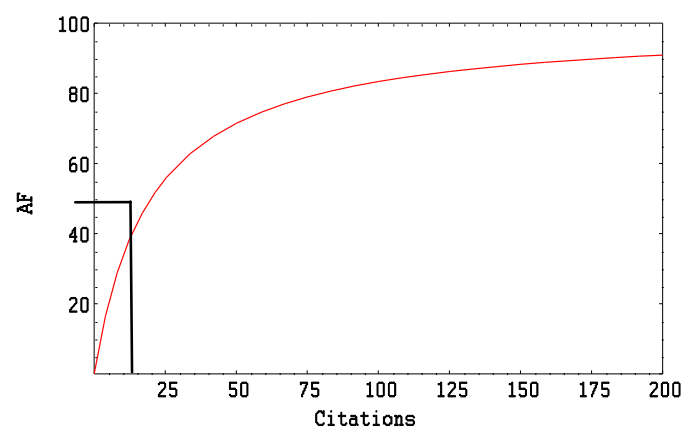

Fig. 1: Plot of $A F$ as a function of the number of citations. The published papers in the preceding year were assumed to be 20 papers.

In Fig. 1 we plot the relation between $A F$ and the number of citations. We fix the number of published papers as 20 papers in the preceding year. It is shown that as the number of citations is increased the $A F$ is increased in agreement with the $I F$. For example if the number of citations is 5 then the $\mathrm{AF}$ is $20 \%$. It is interesting to note that when the number of citations is increased dramatically from the number of published papers in the preceding year, the $A F$ increases by very small values. This means that, this new factor is very sensitive when the number of published papers in preceding year of a journal is close to or less than the number of citations.

Table 1

\begin{tabular}{|l|l|l|l|l|}
\hline Citations & 5 & 10 & 20 & 40 \\
\hline$A F$ & 20 & 33.3 & 50 & 66.6 \\
\hline
\end{tabular}

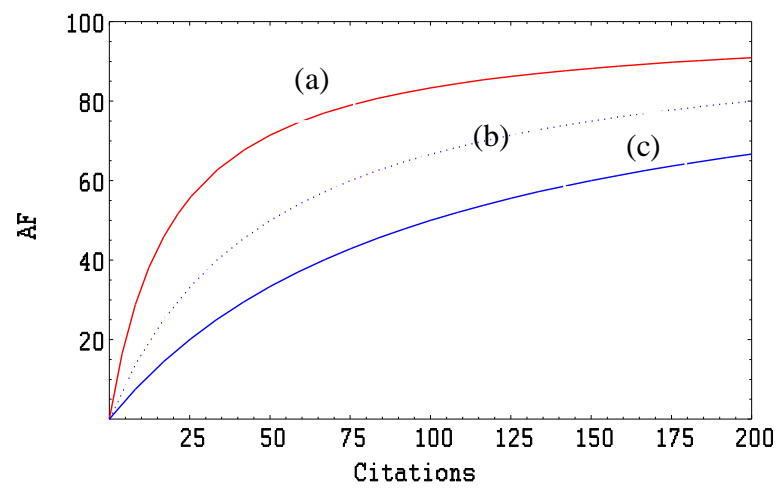

Fig. 2: Example of three different journals, with different numbers of published papers $(\mathrm{N})$ in the presiding year, where (a) $\mathrm{N}=20$, (b) $\mathrm{N}=50$ and (c) $\mathrm{N}=100$.

From Fig. 2, it is shown that $A F$ decreases if the number of published papers in a journal is increased with a fixed value of citations' number.

As for a given individual I suggest to use a modified version of the Hirsch index as follows:

$$
A s F=\left(\frac{h}{h+1}\right) \times 100 \text {. }
$$


From this equation it is shown that $A s F$ is an increasing number according to the increasing of $h$ but does not exceed 100. In particular, assume that the researcher has $h$-index 1 this corresponds to $A s F=50 \%$ and h-index 5 corresponds to $A s F=83.3 \backslash \%$ and so on. When $h$ index gets larger the difference in $A s F$ became smaller (see table 2)

Table 2

\begin{tabular}{|c|c|c|c|c|c|c|}
\hline$h$-index & 1 & 5 & 10 & 20 & 40 & 100 \\
\hline$A s F$ & 50 & 83.3 & 90.9 & 96.2 & 97.6 & 99 \\
\hline
\end{tabular}

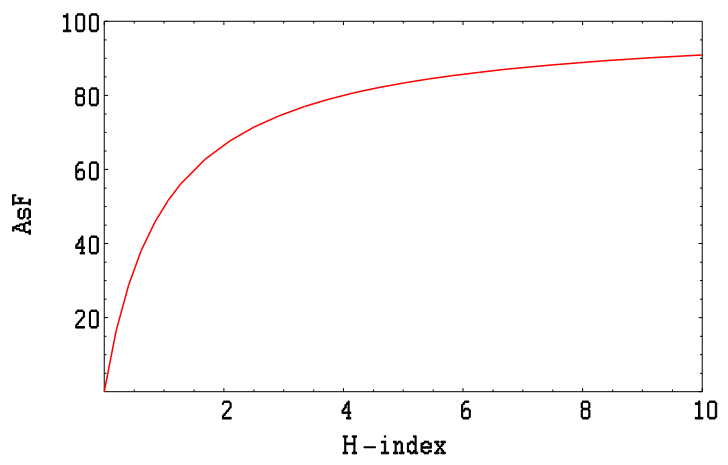

Fig. 3: Plot of $A s F$ as a function of the $h$-index.

In conclusion, we have shown that the suggested AF independently gives a direct indication of any journal rank. Also, the suggested model, $A s F$, for estimating the impact of the scientist's cumulative research contributions gives a direct percentage that can be used to compare different individuals. Finally, we showed that the simultaneous growth of $A F(A s F)$ is dependent on the growth of $I F$ ( $h$-index).

\section{Acknowledgments:}

I would like to thank, W. Alnaser, Peter Leach, Arthur Mc-Gurn, A.-S. F. Obada, A Al-Hasheme, and K. Al-Khalili for helpful discussions. Also, I would like to thank the referees for constructive comments.

\section{References}

[1] Introducing the Impact Factor, Retrieved 2009-08-26.

[2] JCR with Eigenfactor, Retrieved 2009-08-26.

[3] Web of Knowledge, Real Facts, Quality and Quantity. Retrieved 2010-05-05.

[4] J.E. Hirsch, An index to quantify an individual's scientific research output, Proc. Nat. Acad. Sci. 46, 16569 (2005)

[5] www.scopus.com

[6] Leo Egghe, Theory and practice of the g-index, Scientometrics, 69, 131 (2006). 